\title{
Detected, yet not Diagnosed - Breast Cancer Screening with MRI Mammography in High-Risk Women
}

\author{
Joerg Heil ${ }^{* *}$ Elena Czink ${ }^{a *}$ Anne Schipp ${ }^{\mathrm{b}}$ Christof Sohn $^{\mathrm{a}}$ \\ Hans Junkermann ${ }^{\mathrm{a}}$ Michael Golatta ${ }^{\mathrm{a}}$ \\ aUniversity Breast Unit,

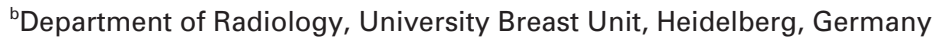

\section{Keywords}

Breast imaging - Familiar breast cancer .

Double reading

\section{Summary}

Background: MRI has been demonstrated to be the most sensitive imaging method for detecting breast cancer in women at high risk, allowing depiction of cancers that are occult on mammography, ultrasound and clinical breast examination. This high sensitivity is tempered by imperfect specificity due to overlap in the features of benign and malignant lesions. Case: We present the case of a young BRCA2 mutation carrier whose breast cancer could have been diagnosed 2 years earlier; this is a rare case of a false-negative finding in MRI. Discussion: We discuss morphological, physiological and psychological reasons for underestimation of MRI sets, especially in young women. Conclusion: We conclude that double reading in MR screening for breast cancer in high-risk women, as conducted for mammography screening, could be considered.

\section{Introduction}

The annual breast MRI (magnetic resonance imaging) is an integral part of the intensified early detection program for women at high risk for breast cancer. In Germany, the high-

*Both authors contributed equally to this article.

\section{KARGER}

Fax +497614520714

Information@Karger.de

www.karger.com

\section{(C) 2012 S. Karger GmbH, Freiburg}

$1661-3791 / 12 / 0073-0236 \$ 38.00 / 0$

Accessible online at:

www.karger.com/brc

\author{
Schlüsselwörter \\ Bildgebung Mamma · Hochrisikokollektiv . \\ Doppelbefundung
}

\section{Zusammenfassung}

Hintergrund: Die Magnetresonanztomographie ist das Verfahren mit der größten Sensitivität in der Früherkennung von Brustkrebs bei Hochrisikopatientinnen. Es erlaubt eine frühe Darstellung von malignen Läsionen, die mit Mammographie, Ultraschall oder der klinischen Untersuchung nicht erkannt werden können. Diese hohe Sensitivität wird jedoch durch die geringere Spezifität eingeschränkt, die auf Überlappungen der Charakteristika für gut- und bösartige Läsionen beruht. Fallvorstellung: Wir stellen mit dem Fall einer jungen Patientin, die Trägerin einer BRCA2-Mutation ist, den seltenen Fall der Unterschätzung der MRT, somit der falsch negativen Beurteilung, vor. Diskussion: Im Folgenden besprechen wir morphologische, physiologische und psychologische Gründe für eine mögliche Unterschätzung der MRT-Aufnahmen, vor allem bei jungen Frauen. Schlussfolgerung: Hieraus lässt sich die Frage ableiten, ob eine Doppelbefundung der MRT-Bilder, wie sie bereits für das Mammographie-Screening Standard ist, eingeführt werden könnte.

risk screening program is based at specialised centres and applies to women aged 25-55 years who fulfil the high-risk criteria of the German Consortium for Hereditary Breast and Ovarian Cancer (GCHBOC) [1]. MRI provides an ostensibly good sensitivity but in clinical routine the low specificity always challenges this diagnostic tool - most commonly, it results in overestimation of the findings. The presented case demonstrates that breast MRI may also be underestimated. 


\section{Case Report}

\section{Overview}

The patient, at the time of diagnosis 37 years old, is a carrier of a BRCA2 gene mutation. She first presented herself at the breast unit at the University Hospital Heidelberg in November 2004 in line with the high-risk screening program for breast cancer. Since then, she has regularly passed through the recommended examinations. In November 2009 she was diagnosed with asymptomatic breast cancer.

\section{MRI Technique}

Diagnostic breast MRI was performed using a 1.5 Tesla field scanner (Avanto ${ }^{\circledR}$; Siemens Medical, Erlangen, Germany) and a bilateral breast coil (7-channel Biopsy Breast Array; Invivo, Orlando, USA). The investigation was performed in a prone position using a slight compression of the breast. First, an axial T2-weighted Turbo-Spin-Echo (TSE) sequence was acquired. Next, an axial T2-weighted Turbo-Inversion-RecoveryMagnitude (TIRM) sequence was performed. Subsequently, the dynamic part of the investigation was started: an axial T1-weighted flash 3D gradient-echo sequence was repeated 7 times. At the beginning of the second axial T1-weighted flash 3D sequence the contrast medium was started (Gadovist, Bayer Schering Pharma, Berlin, Germany). A subtraction of the images was performed, subtracting the contrast enhanced images of a sequence from the native images. Finally, a contrast-enhanced axial T1weighted flash $3 \mathrm{D}$ fat-saturated gradient-echo sequence was performed. For the analysis, the images were sent to a separate CAD system (Dynacad, Invivo Inc., Gainesville, FL, USA). The images were interpreted by radiologists experienced in breast diagnostics (more than 100 MRIs per year) applying the BI-RADS classification for a standardised description of the images [2].

\section{Imaging}

In 2004 and early 2006, mammography, ultrasound and MRI did not show any suspicious findings (BIRADS 2 and ACR IV on both sides). In December 2006, 11 months later, the MRI showed a laminar contrast medium enhancement on both sides dominating on the exterior parts of the breasts (BIRADS 3 on both sides), without single lesions being detectable. Due to diagnostic uncertainty caused by the laminar contrast medium enhancement, the radiologists recommended repeating the examination 6 months later and suggested discontinuing the use of oral contraceptives 1 month prior to the planned examination. In July 2007, a distinct reduction of contrast medium enhancement was found. At the next imaging appointment, in September 2008, no suspicious lesions were observed, and morphological and perfusion criteria were negative. The simultaneously conducted mammography and ultrasound examinations did not display any signs of malignancy. The gland tissue appeared dense on both imaging techniques (ACR IV); the mammography showed foci of microcalcifications interpreted as adenosis of the breast (BIRADS 2). In September 2009, the MRI showed a suspicious lesion of $6 \times 7 \mathrm{~mm}$ on the left side, which was located centrally, close to the pectoralis muscle (fig. 1). The lesion presented a type 1 curve, which was assessed BIRADS IV.

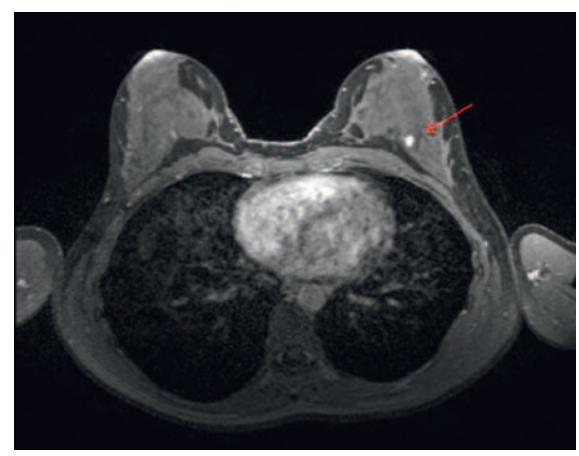

Fig. 1. MRI 2009:

Small lesion in the left breast $(6 \times 7 \mathrm{~mm})$.
Thereupon, mammography and ultrasound were performed, but could, in the first instance, not reproduce the finding. With regard to the dubious MRI finding a vacuum biopsy was recommended to exclude malignancy. As the lesion was not accessible for MRI-directed biopsy due to its location next to the pectoral muscle, the lesion was located by an O-twist marker using MR guidance. Consecutively, a stereotactic vacuum biopsy of the area was performed and histology revealed an invasive ductal carcinoma of the breast (G 2, Ki-67: 5\%, oestrogen receptor $100 \%$, progesterone receptor $100 \%$, Her2neu negative).

In retrospective analysis of the MRI examinations, the lesion could also be identified in the imaging sets of September 2008 and July 2007, whereupon, with the course of time, a progress in size from $3 \times 6 \mathrm{~mm}$ in September 2008 to $6 \times 7 \mathrm{~mm}$ in September 2009 was observed (fig. 2). Ultimately, the patient suffered from a carcinoma of the left breast, stage pT1b pN0.

\section{Discussion}

This case addresses the question of whether the diagnosis could have possibly been made earlier, as the lesion was, retrospectively, identified on prior imaging sets. Frequent over- and rare underestimation of dubious lesions in MRI analysis represent the dilemma of breast cancer screening and pose a challenging topic to the examiner.

When reflecting on MRI as a diagnostic tool for early detection of breast cancer, one of the most frequently addressed topics is its lack of specificity. In the literature, specificity ranges from 59.4 to $95 \%$, depending on the study population and experience of the institution reporting the data [3]. However, breast MRI does provide an additional benefit with respect to sensitivity in screening BRCA1 and BRCA2 mutation carriers and non-BRCA women at high risk for breast cancer, as a Dutch trial with a high-risk collective demonstrated [4]. The often criticised low specificity of MRI does not play such a preponderant role in the high-risk collective due to the high prevalence of breast cancer in this cohort [5] even though it still ranges between 81 [6] and 89\% [7] according to the British and Dutch high-risk collective trials. Additionally, according to Kriege et al. [4], the rate of false-positive MRI findings varies in the group of BRCA mutation carriers. They observed a higher rate in premenopausal women than in postmenopausal women $(10.7$ vs. $6.2 \%, \mathrm{p}<0.001)$, along with a greater probability of false-positive findings in women with a high breast density compared to women with a

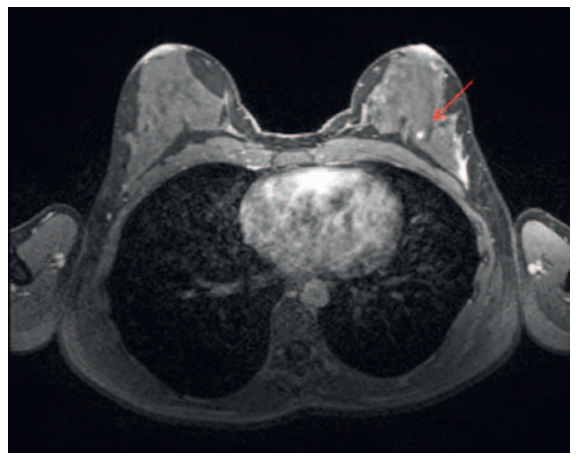

Fig. 2. MRI 2008

Small lesion in the left breast $(3 \times 6 \mathrm{~mm})$. 
low breast density $(11.3$ vs. $8.9 \%, p=0.01)$. In line with these findings, overestimation could have been considered probable in our case, as the patient is premenopausal and presented a density of ACR IV on both sides. It is generally possible that physicians who evaluate the MRI data may be influenced by the dogma of overestimation of MRI in such a way that they may tend to underestimate non-distinctive contrast medium enhancements.

Obdejin et al. [8] conducted a trial to examine the reasons for false-negative MRI findings. The trial involved 22 patients from the Dutch high-risk cohort with negative MRI findings but histologically proven breast cancer. In 12 cases, even the review of the MRI sets remained unobtrusive; in 8 of these cases the patients suffered from a small DCIS (ductal carcinoma in situ) that was eventually diagnosed by mammography. These findings may suggest a possible weakness of MRI concerning this subtype [7], even though the latest publications indicate a high sensitivity of MRI in detecting DCIS [911]. The diagnosis is achieved on the basis of neovascularisation and asymmetric contrast medium enhancement [12]. Among the 22 cases of the above-mentioned analysis [8], 9 cases had lesions that could be detected on reviewing the MRI sets; the authors attributed this misinterpretation to the underestimation of the contrast medium dynamics and the connected malignancy criteria. For 2 patients wide laminar and diffuse contrast medium enhancements complicated the evaluation of the breast; even in the review no malignant lesions could be identified. Such a laminar contrast medium enhancement also occurred in the patient of this case, especially in the area where the lesion was later detected, and this may have disguised the lesion in earlier imaging sets.

Laminar contrast medium enhancements and other foci with suspicious contrast medium dynamics can occur during the menstrual cycle of healthy women [13]. They usually originate in the follicular phase, as the histamine-like effect of oestrogen enhances the permeability of the vessels prior to ovulation and, therefore, causes a higher contrast medium uptake. In the secretory phase, the enhanced mitotic activity and the rising progesterone level cause similar effects. Therefore, the authors recommend setting the MRI appointment between days 3 and 14 of the menstrual cycle, when extraction flow product and signal enhancement are lowest. This stands in slight contrast to previous studies where enhancement was reported to be lowest on days 7-20 [14] and 7-14 [15], respectively. Consequently, as a general recommendation, screening appointments should be set in the second week of the menstrual cycle, as this period is approved by all authors [13-15].
The diagnostic dilemma of over- and underestimation of foci with non-distinctive contrast medium dynamics has to be acknowledged by every examiner, who should, in cases of uncertainty, seek a second opinion. In general, the screening of high-risk patients as a method of early detection should only be administered at accredited centres with experienced examiners.

To obtain optimal results one could argue for a quality management program, complementing the high-risk program for breast cancer (similar to the current German mammography screening). In mammography screenings, the independent double reading by 2 experienced examiners, finally reaching a consensus, improved sensitivity without compromising specificity [16]. This procedure should also be tested for its cost efficiency in the high-risk program for breast cancer.

Despite all of these remarks, it has to be noted that an advanced diagnosis would probably not have altered the patient's therapy scheme and prognosis. According to the College of American Pathologists Consensus Statement of 1999 [17], the 10-year-survival rate is $90 \%$ for a tumour size smaller than $1 \mathrm{~cm}$ and a negative lymph node status, which was the case at the hypothetically further advanced tumour at the actual time of diagnosis. Therefore one can assume that, with respect to prognosis, the delayed diagnosis did not have disadvantageous results for the patient.

\section{Conclusion}

MRI mammography is a valuable and important method in screening programs for high-risk women as its combination with traditional imaging techniques entails a benefit in sensitivity. Although the examiners are warned about the limited specificity and the consequent overestimation, one is not automatically protected from underestimation. In particular, the evaluation of the imaging sets in young premenopausal women with dense breasts remains a challenging topic.

The interpretation of MRI data requires a lot of experience and should be effected in specialised and accredited centres. Implementing a quality management program including standardized double reading might help to meet the aim of optimizing high-risk screening programs.

\section{Disclosure Statement}

The authors declare no conflicts of interest.

\section{References}

$\checkmark 1$ Schmutzler R, Schlegelberger B, Meindl A, et al.: Counselling, genetic testing and prevention in women with hereditary breast- and ovarian cancer. Interdisciplinary recommendations of the con- sortium 'Hereditary Breast- and Ovarian Cancer' of the German Cancer AiD. Zentralbl Gynakol 2003;125:494-506.
2 ACR Guidelines and Standards Committee, L W Basset, W A Berg, et al.: ACR practice guideline for the performance of contrast-enhanced magnetic resoonanc imaging (MRI) of the breast. 
2008 cited 2011 January; Available from: www.acr.org/SecondaryMainMenuCategories/quality_safety/guidelines/breast/mri_breast.aspx.

3 Ravert PK, Huffaker C: Breast cancer screening in women: An integrative literature review. J Am Acad Nurse Pract 2010;22:668-673.

4 Kriege M, Brekelmans CT, Obdeijn IM, et al.: Factors affecting sensitivity and specificity of screening mammography and MRI in women with an inherited risk for breast cancer. Breast Cancer Res Treat 2006;100:109-119.

5 Junkermann H: Mammadiagnostik zur Brustkrebsfrüherkennung. Onkopipeline 2009;2:146-152.

6 Leach MO, Boggis CR, Dixon AK, et al.: Screening with magnetic resonance imaging and mammography of a UK population at high familial risk of breast cancer: a prospective multicentre cohort study (MARIBS). Lancet 2005;365:1769-1778.

7 Kriege M, Brekelmans CT, Boetes C, et al.: Efficacy of MRI and mammography for breast-cancer screening in women with a familial or genetic predisposition. N Engl J Med 2004;351:427-437.

8 Obdeijn IM, Loo CE, Rijnsburger AJ, et al.: Assessment of false-negative cases of breast MR imaging in women with a familial or genetic predisposition. Breast Cancer Res Treat 2010;119:399-407.

$\checkmark 9$ Kuhl CK, Schrading S, Bieling HB, et al.: MRI for diagnosis of pure ductal carcinoma in situ: a prospective observational study. Lancet 2007;370:485-492.

10 Berg WA, Gutierrez L, NessAiver MS, et al.: Diagnostic accuracy of mammography, clinical examination, US, and MR imaging in preoperative assessment of breast cancer. Radiology 2004;233:830-849.

11 Warner E, Plewes DB, Hill KA, et al.: Surveillance of BRCA1 and BRCA2 mutation carriers with magnetic resonance imaging, ultrasound, mammography, and clinical breast examination. JAMA 2004;292:1317-1325.

12 Cao Y, Paner GP, Kahn LB, et al.: Noninvasive carcinoma of the breast: angiogenesis and cell proliferation. Arch Pathol Lab Med 2004;128:893-896.

13 Delille JP, Slanetz PJ, Yeh ED, et al.: Physiologic changes in breast magnetic resonance imaging during the menstrual cycle: perfusion imaging, signal enhancement, and influence of the T1 relaxation time of breast tissue. Breast J 2005;11:236-241.

14 Muller-Schimpfle M, Ohmenhauser K, Stoll P, et al.: Menstrual cycle and age: influence on parenchymal contrast medium enhancement in MR imaging of the breast. Radiology 1997;203:145-149.

$\checkmark 15$ Kuhl CK, Bieling HB, Gieseke J, et al.: Healthy premenopausal breast parenchyma in dynamic contrast-enhanced MR imaging of the breast: Normal contrast medium enhancement and cyclical-phase dependency. Radiology 1997; 203:137-144.

16 Duijm LE, Louwman MW, Groenewoud JH, et al.: Inter-observer variability in mammography screening and effect of type and number of readers on screening outcome. Br J Cancer 2009;100:901-907.

17 Fitzgibbons PL, Page DL, Weaver D, et al.: Prognostic factors in breast cancer. College of American Pathologists Consensus Statement 1999. Arch Pathol Lab Med 2000;124:966-978. 